\title{
A pH/glutathione double responsive drug delivery system using molecular imprint technique for drug loading
}

Kelin Zhang a , Xiujuan Guan ${ }^{\mathrm{a}}$, Yanxin Qiu ${ }^{\mathrm{b}}$, Dongdong Wang ${ }^{\mathrm{a}}$, Xiaoyu Zhang ${ }^{\mathrm{b}}$, Haixia Zhang *a

${ }^{a}$ College of Chemistry and Chemical Engineering, Key Laboratory of Nonferrous Metal Chemistry and Resources Utilization of Gansu Province, Lanzhou University, Lanzhou 730000, China

'Institute of Physiology, School of Basic Medical Sciences, Lanzhou University, Lanzhou 730000, China

\begin{abstract}
A surface molecular imprinting polymer (SMIP) with doxorubicin (DOX) as the template was prepared on the surface of mesoporous silica nanoparticles (MSNs), which were further used as DOX carriers. The loading amount of DOX was calculated as $10.5 \pm 0.2 \mathrm{wt} \%$ with loading efficiency of $70 \pm 8 \%$. The DOX release was controlled because the monomer molecule used in polymerization of SMIP containing sulfur-sulfur bonding, which could be decomposed with an acidic $\mathrm{pH}$ and glutathione (GSH). Under an acidic $\mathrm{pH}$ and high concentration of $\mathrm{GSH}$, there was greater release of DOX than under normal physiological conditions, which induced less damage to normal cells than to cancer cells. Confocal laser scanning microscopy studies verified the invasion of the DOX within SMIP into TCA8113 cancer cells. These results indicate that the prepared SMIP was an effective nanocarrier.
\end{abstract}

Keywords: molecular imprint polymer, nanocarrier, control release

\section{Introduction}

Stable mesoporous silica nanoparticles (MSNs) with high surface area and pore are excellent candidates as versatile drug carriers for controlled release owing to the capacity of their pore channels to adsorb drugs [1]. Easy chemical modifications of their surface or combinations with magnetic and/or luminescent compounds enrich their application for achieving sustained drug release [2-4].

High drug loading is important for a controlled drug delivery system. Carriazo et 
al [5] discovered that the drug release rate decreased with increasing drug content because the solvents had difficulty penetrating into the pore channels, thus preventing drug from leaching out of the carriers. Normally, drug loading involved physical adsorption [6], solvent evaporation [7] and covalent linkage with drug carriers [8]. The majority of studies have focused on drug release rather than drug loading [9-10].

The molecular imprinting technique (MIT) [11-12] is based on the development of an interaction between a template molecule and suitable functional monomers during the pre-polymerization step and then achieving the polymerization through an initiator. The cross-linked polymer can be used as an effective adsorbent after the template is removed. MIT is a well developed tool in the analytical field [13-14] and molecularly imprinted polymers (MIPs) have recently been used to develop drug delivery systems [15-21]. In these works, the authors prepared MIPs, removed the template (drug) to form caves, and loaded drugs on blank MIP again. This way of drug loading has two shortages: one is needing a long time to carried out the adsorption and the other is that not all the caves can be used effectively to adsorb the drug because it is difficult for the drug molecules to enter the caves far from the surface of MIP owe to mass transfer resistance.

Removing the template is a necessary but time-consuming step in MIT when MIP used as special adsorbent; it can take $24 \mathrm{~h}$ with an intensive soxhlet extraction [22] with the adventure of residual template molecules left in the MIP. Given that, is it possible to drugload using MIT? One possibility is to use the drug as template during the polymerization step of MIP. After the preparation, the template does not need to be removed and the MIP is used as the delivery system directly. Lacking the steps of template removal and drug loading, the less time is used but the highest loading efficiency obtained .

In the present study, the MIT technique was used to load drug for the first time. MSN particles, $50 \mathrm{~nm}$ in size, were fabricated, and then MIP with doxorubicin (DOX) as template was synthesized on the surface of MSNs. During the polymerization, DOX was encapsulated in the polymer to achieve loading. Moreover, MABHD [23], a monomer containing disulfide bond, was home- made and used in the MIP procedure. 
This monomer was degraded with high glutathione (GSH) concentration and acidic conditions for controlled release of DOX.

\section{Experimental}

\subsection{Reagents and Materials}

Ethanol $\left(\mathrm{C}_{2} \mathrm{H}_{5} \mathrm{OH}, 99.7 \%\right)$ and concentrated ammonia solution $\left(\mathrm{NH}_{3} \cdot \mathrm{H}_{2} \mathrm{O}\right.$, 25\%-28\%) were purchased from Tianjin Guangfu Fine Chemical Research Institute (Tianjin, China). Tetrahydrofuran (THF, 99.5\%) and triethylamine (TEA, 99\%) purchased from Tianjin Chemical Reagent (Tianjin, China) were used after being stirred overnight over $\mathrm{CaH}_{2}$ and distilled under reduced pressure. (3-aminopropyl) trimethoxysilane (APTMS, 97\%), dimethyl aminopyridine (DMAP) and ethylene glycol dimethacrylate (EGDMA) were supplied by Alfa Aesar (Tianjin, China). EGDMA was distilled before used. Azo-bis-isobutyronitrile (AIBN) was obtained from Yuefeng Chemical Company (Tianjin, China). Bis (trichloromethyl) carbonate (BTC) was from Chaofan Company (Lianyungang, China). Glutathione (GSH, 98\%) were purchased from Aladdin Chemistry Co., Ltd. (Beijing, China). Doxorubicin (DOX) was obtained from Huafeng United Technology CO. Ltd. (Beijing, China). Deionized (D.I.) water was prepared from Millipore (Bedford, MA, America). All these reagents were of analytical grades.

\subsection{Characterization}

Transmission electron microscopy (TEM) micrographs were carried out on a JEM-1200EX TEM (JEOL, Japan) using the copper grid as the sample holder, operating at an accelerated voltage of $100 \mathrm{kV}$. The size distribution of materials were determined by dynamic light scattering (DLS) using a BI-200SM (Brookhaven, USA) with angle detection at $90^{\circ}$. The Fourier transform infrared (FTIR) spectra were acquired with a Nicolet 20 NEXUS 670 FTIR spectrophotometer (Ramsey, MA, USA) using KBr pellets. Thermo gravimetric analysis (TGA) was taken on a STA PT1600 Thermal Analyzer Instruments (Linseis, Germany) with the heating rate of $10^{\circ} \mathrm{C} \mathrm{min}^{-1}$ under $\mathrm{N}_{2}$ atmosphere. The synthesis products were determined by elementary analysis 
using a Vario EL elemental analysis system (Elementar, Germany). Nitrogen adsorption-desorption measurements were performed at $77 \mathrm{~K}$ on a Tristar 3000 Surface Area and Porosimetry analyzer (Micromeritics Instrument Corp., USA). The surface-area measurement was based on the Brunauer-Emmett-Teller (BET) method, and the pore-size distribution was based on the Barrett-Joyner-Halenda (BJH) formula.

Fluorescence spectra were recorded with a RF-5301PC fluorescence spectrometer (Shimadzu, Japan). The cellular images were acquired with a confocal laser scanning microscope (CLSM, ZEISS, LSM 510 Meta, Germany).

\subsection{Synthesis of molecular imprint nanocarrier for doxorubicin}

The MABHD (Fig S1, Supplementary information) used in MIT was synthesized according to previous work in our lab [23]. MSNs were synthesized according to the work of Pan [24].

\subsubsection{Synthesis of amino modified MSN}

MSNs modified to have an $\mathrm{NH}_{2}$ group were synthesized as following: MSNs (100 mg) were dispersed using ultrasound in $50 \mathrm{~mL}$ of ethanol. To which $200 \mu \mathrm{L}$ of APTMS and $100 \mu \mathrm{L}$ of concentrated ammonium solution were added. After refluxing for $24 \mathrm{~h}, \mathrm{MSNs}-\mathrm{NH}_{2}$ products were centrifuged and dried in vacuum at $60{ }^{\circ} \mathrm{C}$.

\subsubsection{Synthesis of MABHD-modified MSNs}

DMAP (156 mg) and MABHD (100 mg) were dispersed in $20 \mathrm{~mL}$ of THF, and $\mathrm{N}_{2}$ was gassed into the solution for $15 \mathrm{~min}$. BTC (42 mg) was added, and the dispersion was stirred continuously at room temperature for $30 \mathrm{~min}$. $\mathrm{MSNs}^{-\mathrm{NH}_{2}}$ (70 mg) were added and stirred for another $6 \mathrm{~h}$. The product, MSN@MABHD, was centrifuged and washed with ethanol three times.

\subsubsection{Synthesis of nanocarrier}

DOX (6 mg) and MSNs@MABHD (10 mg)were dispersed in $10 \mathrm{~mL}$ of ethanol, and the mixture was stirred continuously in the dark for $24 \mathrm{~h}$. Subsequently, EGDMA $(60 \mu \mathrm{L})$ was added to the mixture and stirred for $30 \mathrm{~min}$ in an ice bath. AIBN (10 mg) 
was then added as an initiator, and the mixture was gassed with $\mathrm{N}_{2}$ for $10 \mathrm{~min}$. The gassed mixture was stirred in container at $60{ }^{\circ} \mathrm{C}$ for $24 \mathrm{~h}$. The final product MSNs@DOX@MIP was obtained after centrifugation and washing with ethanol.

The material, MSNs @NIP, was prepared using the same method except without adding DOX.

\subsection{Drug release}

MSNs@DOX@MIP (1.5 mg) was added to $3 \mathrm{~mL}$ of phosphate buffer solutions (PBS) at $\mathrm{pH} 7.4$, or $\mathrm{pH} 5.0$, and or the same PBS plus $10 \mathrm{mM} \mathrm{GSH}$ in dialysis bag (cut off molecular weight $3500 \mathrm{Da}$ ). The outside of the dialysis bag was $40 \mathrm{~mL}$ of same media. The dialysis experiment was continued in water bath at $37{ }^{\circ} \mathrm{C}$ with gentle shaking. At specific time intervals, $3 \mathrm{~mL}$ of release media was removed and replenished with an equal volume of fresh medium. Finally, the released DOX was calculated by measuring the fluorescent spectrometry signal of DOX (excitation at $488 \mathrm{~nm}$ and emission at $586 \mathrm{~nm}$ ). The drug release studies were performed in triplicate for each sample.

\subsection{Toxicity assessment}

The human tongue squamous cell carcinoma TCA8113 cell lines was provided by the Biology Preservation Center of the Shanghai Institute of Materia Medica and maintained with RPMI 1640 medium containing $10 \%$ fetal bovine serum (FBS), penicillin $\left(100 \mathrm{U} \mathrm{mL}^{-1}\right)$, and streptomycin $(100 \mu \mathrm{g} \mathrm{mL}-1)$ at $37{ }^{\circ} \mathrm{C}$ in a humidified atmosphere with $5 \% \quad \mathrm{CO}_{2}$. The in vitro cytotoxicity of MSNs@DOX@MIP, MSNs@NIP and DOX were evaluated by sulforhodamine B (SRB) assays.

TCA8113 cells were seeded in a 96 -well plate $\left(3.0 \times 10^{4}\right.$ cells per well $)$ overnight. Culture medium was replaced with fresh medium containing the above three samples at concentrations that ranged from 0.16 to $200 \mu \mathrm{g} \mathrm{mL} \mathrm{L}^{-1}$ for $24 \mathrm{~h}$, respectively. Cells were then fixed by the addition of cold $10 \%$ trichloroacetic acid (TCA;100 $\mu \mathrm{L}$ at $4{ }^{\circ} \mathrm{C}$ ) for $1 \mathrm{~h}$ in each well. The wells were gently washed five times with deionized water and then stained with $0.4 \%$ SRB solution $(100 \mu \mathrm{L}$ per well) for $30 \mathrm{~min}$ at room temperature. The unbound SRB was rinsed away with $1 \%$ acetic acid. As the plate 
air-dried again, aqueous tris base (tris(hydroxymethyl)aminomethane) (150 $\mu \mathrm{L}, 10$ $\mathrm{mM}$ ) was added into each well to solubilize the SRB dye. The absorbance values were read on a microplate reader (Huake company, Shanghai, China) at $570 \mathrm{~nm}$. This procedure was repeated three times, with the cells cultured in the media without adding the materials as control.

\subsection{Cellular uptake studies}

The ability of MSNs@DOX@MIP nanoparticles to enter cancer cells was observed using the confocal laser scanning microscope. The TCA8113 cells $\left(3.0 \times 10^{4}\right.$ cells per well) were seeded in a six-well plate at $37{ }^{\circ} \mathrm{C}$ overnight. Free DOX and MSNs@DOX@MIP (at $10 \mu \mathrm{g}$ DOX per mL) were then added, respectively. Cell nuclei were stained using 4,6-diamidino-2-phenylindole (DAPI) (Sigma Aldrich). After a further 3 or $24 \mathrm{~h}$ of incubation, the cells were washed with PBS three times to remove the dead cells and the drugs adsorbed on the outer surface of the cell membrane. The MSNs@DOX@MIP uptake was visualized at an excitation wavelength of $488 \mathrm{~nm}$.

\section{Results and discussion}

\subsection{Characterization}

Fig. 1 shows the procedure for synthesizing MSN@DOX@MIP. MSN is a sorbent with superior capability and DOX can adsorb into the pores of MSNs by physical adsorption before the polymerization [25]. After polymerization, DOX was fixed in the polymer because it combined with MABHD monomer and amino groups on MSN[26]. Results of the element analysis used to trace the synthesis are shown in

Table 1. The presence of nitrogen denotes the MSN is modified with amino group in step 1 and the sulfur element denotes the MABHD group is modified on amino MSN material. The changed carbon amount denotes the successful polymerization in step 3. According to the molar ratio of $\mathrm{N}$ and $\mathrm{S}$ element, it can be calculated that one fifth of amino groups react with MABHD, which means that both the amino group on MSN and the double bond in MABHD act as the monomers for MIP synthesis. 
Fluorescence spectra were used to assess the polymerization of MIP and NIP. MSN@DOX@MIP and DOX had similar spectra but the MSN@MABHD and MSN @NIP had no fluorescence. DOX was successfully mixed in the MSN@DOX@MIP (Fig. 2).

TGA was used to study the degradation of the materials. The MSNs and MSNs@MABHD were stable because of the little organic component. MSNs@NIP lost approximately $72 \%$ of their weight as the temperature increased to $425^{\circ} \mathrm{C}$, which indicated that the amount of polymer on the surface of MSNs was adequate (Fig. S2). MIPs occupy near three fourth of the amount of material, which can adsorb DOX efficiently. IR spectra of the materials were compared to further verify if fabrication was successful. (Fig. S3). Compared with the IR spectrum of MSNs (a), the peaks at 1701 and $1632 \mathrm{~cm}^{-1}$ belonged to the stretching vibration of $\mathrm{C}=\mathrm{O}$ and $\mathrm{C}=\mathrm{C}$ in MSNs @MABHD (b) and the peak that appeared at $1731 \mathrm{~cm}^{-1}$ assured that the conjugate bond in MABHD was destroyed and that MSNs @NIP (c) was synthesized successfully.

The average particle size of MSNs was determined by TEM as $47 \mathrm{~nm}$ and DLS as $57 \mathrm{~nm}$. The difference of $10 \mathrm{~nm}$ from the two methods results from the different sample status. One was in solid and the other in solution (Fig. 3). The surface and pore size of MSNs were measured using an $\mathrm{N}_{2}$ absorbance method, and found to be $149 \mathrm{~m}^{2} \mathrm{~g}^{-1}$ and $2.7 \mathrm{~nm}$, respectively (Fig. S4). The size of pore means that MSN is synthesized successfully. After polymerization, the particle size increased to approximately 90 to $100 \mathrm{~nm}$, which was in accordance with the TGA results. The thick MIP layer obtained can assure the larger DOX loading and hinder the burst release of DOX.

\subsection{Drug release from DOX@MSNs@MIP}

Owing to the use of MABHD as the monomer, the S-S bond in MABHD breaks and induces the disassembly of the nanocarrier, facilitating the release of DOX when GSH comes in contact with DOX@MSNs@MIP. Subsequently, the polymer is removed from the surface of MSN, and DOX is further released from the pores of 
MSNs.

The loading capacity of DOX was calculated as $10.5 \pm 0.2 \mathrm{wt} \%$ and the entrapment efficiency was $70 \pm 8 \%$ using MIT, which indicate that polymerization in MIT could be used to drug loading directly. The loading capacity was much higher than the reported (30-40 $\left.\mathrm{mg} \mathrm{g}^{-1}[16] ; 10 \mathrm{mg} \mathrm{g}^{-1}[17]\right)$, in which the drug loading was carried out on the MIP after removal of the template. Moreover, DOX was utilized more completely in our work. The residual DOX in solution was $0.5 \mathrm{mg} \mathrm{mL}^{-1}$ in [17] and $0.18 \mathrm{mg} \mathrm{mL}^{-1}$ in our work when the DOX loading reached the balance. Obviously, less DOX was used for the same loading capacity using our method.

The loading capacity and entrapment efficiency of DOX were calculated using the following expressions:

$$
\text { drug loading capacity \% = } \frac{\text { amount of DOX in MSNs@DOX@MIP }}{\text { amount of MSNs@DOX@MIP }} \times 100 \%
$$

$$
\text { drug entrapment efficiency } \%=\frac{\text { amount of DOX in MSN@DOX@MIP }}{\text { total amount of DOX for loading }} \times 100 \%
$$

The DOX release was evaluated in four solution conditions (Fig. 4). First, in 50 mM PBS (pH 7.4), up to 22\% of DOX was released from DOX@MSNs@MIP in the first $12 \mathrm{~h}$. No obvious release can be found later, with the data indicating a decrease to $18 \%$. The possible reason was re-adsorption on the materials. The DOX release was likely from the sites near the surface of the materials because it happened much easier than from the deeper sites. In addition, the DOX release should be happened first from the physical adsorption. Adding $10 \mathrm{mM}$ GSH to the above solution, accelerated the release of DOX. GSH promoted nearly doubled the release (up to 42\%) owing to the breakage of the S-S bond in DOX@MSNs@MIP. The results indicate the successful design of releasing drug through the breakage of S-S bond. The release rate is slower than from the other MIP drug delivery [16], in which the drug release is up to $80 \%$ within $4 \mathrm{~h}$. As forementioned, after template removal the drug loading begins from the MIP surface and it is difficult for the drug molecules to enter the deep caves, which lead the less loading and easier release. 
Second, in $50 \mathrm{mM}$ PBS (pH 5.0), DOX was released faster than in PBS at pH 7.4. The acidic condition helps degrade the polymer by destroying the H-bond between DOX and the monomers, so the release could be up to 50\%. In the acidic solution with GSH, up to $70 \%$ of DOX was released because of the broken S-S bond. The increase extent caused by GSH was similar with that under $\mathrm{pH}$ 7.4. This means that only GSH and not $\mathrm{pH}$ affects the S-S bond. The MIP as a nanocarrier loaded the drug two ways, i.e., adsorption before polymerization and entrapment during polymerization. This dual loading resulted in more release modes.

The release first happened with the drugs loaded through physical absorption and the drugs at the sites near the surface of MIP, which is about $20 \%$. Then, the release happened with the drugs loaded through the bonding of Van der Waals between the monomers and templates, which is easily controlled by change of $\mathrm{pH}$ value. Third, the further release happened with the drugs hidden the depth of MIP, which is accelerated owing to the breakage of disulfide bond. The multiple release ways let the release ratio higher than the reported, for example, only $40 \%$ drug released from the MIP delivery system [17].

The existence of disulfide bond has another advantage that more DOX would be released in cancer cells than in normal cells because of the higher concentration of GSH in cancer cells.

\subsection{Cell viability assay and cellular uptake}

The MTT assay was used to investigate TCA8113 cell viability after $24 \mathrm{~h}$ incubation with free DOX, blank MSN@NIP, and DOX@MSNs@MIP. As shown in Fig. 5a, the MSN@NIP were no cytotoxicity to TCA8113 cells (cell viability near $100 \%$ ) in the concentration range $0-100 \mu \mathrm{g} \mathrm{mL}$, which indicates excellent biocompatibility. Cell death caused by DOX@MSNs@MIP approached 60\% when the DOX content was $2.5 \mu \mathrm{g} \mathrm{mL} \mathrm{L}^{-1}$. For free DOX, approximately $89 \%$ of cells were killed when the DOX content was $2.5 \mu \mathrm{g} \mathrm{mL} \mathrm{mL}^{-1}$ (Fig. 5b). After increasing the

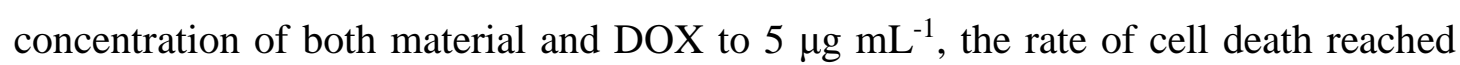
80\% for DOX@MSNs@MIP and 90\% for DOX, both of which were higher release rate than from the silica reported containing MABHD [27]. 
The intracellular delivery of DOX was observed by CLSM images of TCA8113 cells treated with free DOX and DOX@MSNs@MIP after 3 or 24 h incubation, respectively. The CLSM results are shown in Fig. 6. The red fluorescence denotes free DOX or DOX@MSNs@MIP, and the blue fluorescence denotes DAPI-stained cell nuclei. With free DOX as the control (Fig. 6a and 6b), the images of DOX fluorescence suggest that DOX@MSNs@MIP were internalized that the DOX contained in the MSNs was released and entered cell nuclei within $24 \mathrm{~h}$ (Fig. 6d). The rapid, passive diffusion of DOX resulted in stronger DOX fluorescence in TCA8113 cells after incubation for $3 \mathrm{~h}$ (Fig. 6a). However, only weak red fluorescence was observed after incubation with DOX@MSNs@MIP for 3 h (Fig. 6c), which suggests that longer time is needed for nanoparticles to enter cells than when using simple drug molecules. These results confirmed that DOX@MSNs@MIP were able to deliver and release DOX into the nuclei of cancer cells over a long period.

\section{Conclusion}

We report here it is feasible using imprint polymer DOX@MSNs@MIP for drug delivery to cancer cells. The results indicate that loading drugs during the molecular imprint polymerization can lead to multiple release ways to carry out the control release of drugs. DOX was effectively loaded in DOX@MSNs@MIP before and the during polymerization through different mechanisms and delivered to the nuclei of TCA8113 cells via the polymer. This delivery system exhibited efficient $\mathrm{pH}-$ and GSH-controlled release of DOX and excellent biocompatibility. Furthermore, it can save the time of drug loading with higher loading amount. The delivered DOX efficiently inhibit TCA8113 cells growth with the low cytotoxicity of the blank nanocarrier. It deserves to try the method to load other drugs in delivery systems.

\section{Acknowledgements}

This work was financially supported by the National Science Foundation of China (No. 21575055, 21375052). 


\section{References}

[1] J. Lu, H. Mekaru, F. Tamanoi, Development of Mesoporous Silica-based Nanoparticles with Controlled Release Capability for Cancer Therapy, Adv. Drug Delivery Rev. 95(2015)40-49.

[2] L. Sun, Y. Wang, T. Jiang, X. Zheng, J. Zhang, Sun J, Novel chitosan-functionalized spherical nanosilica matrix as an oral sustained drug delivery system for poorly water-soluble drug carvedilol, ACS Appl. Mater. Interfaces. 5(2012) 103-113.

[3] Q. Gan, X. Lu, Y. Yuan, J. Qian, H. Zhou, A magnetic, reversible pH-responsive nanogated ensemble based on $\mathrm{Fe}_{3} \mathrm{O}_{4}$ nanoparticles-capped mesoporous silica. Biomaterials. 32(2011)1932-1942.

[4] L. Zhang, Y. Li, Z. Jin, J. Yu, K. Chan, An NIR-triggered and thermally responsive drug delivery platform through DNA/copper sulfide gates, Nanoscale. 7(2015)12614-12624.

[5] D. Carriazo, M. Del Arco, A. Fernández, C. Martín, V. Rives, Inclusion and release of fenbufen in mesoporous silica, J Pharm Sci. 99(2010)3372-3380.

[6] Y. Fu, X. Li, C. Sun, Z. Ren, W. Weng, C. Mao,and G. Han, pH-Triggered SrTiO3:Er Nanofibers with Optically Monitored and Controlled Drug Delivery Functionality, ACS Appl. Mater. Interfaces.7(2015) 25514-25521.

[7] L. Chi, D. Wu, Z. Li, M. Zhang, H. Liu, C. Wang, S. Gui, M. Geng, H. Li, J.Zhang, Modified Release and Improved Stability of Unstable BCS II Drug by Using Cyclodextrin Complex as Carrier To Remotely Load Drug into Niosomes, Mol. Pharmaceutics. 13(2016)113-121.

[8] D. Yang, W. Chen, J. Hu, Design of Controlled Drug Delivery System Based on Disulfide Cleavage Trigger, J. Phys. Chem. B, 118(2014)12311-12317.

[9] M. Ghasemnejad, E. Ahmadi, Z. Mohamadnia,, A. Doustgani, S. Hashemikia, Functionalized silica nanoparticles as a carrier for Betamethasone Sodium Phosphate: Drug release study and statistical optimization of drug loading by response surface method, Mater. Sci. Eng., C. 56 (2015) 223-232. 
[10] Y. Li, T. Wang, J. Wang, T. Jiang, G. Chen, S. Wang, Functional and unmodified MWNTs for delivery of the water-insoluble drug Carvedilol - A drug-loading mechanism, Appl. Surf. Sci. 257(2011)5663-5670.

[11] J. Wackerlig and R. Schirhag, Applications of Molecularly Imprinted Polymer Nanoparticles and Their Advances toward Industrial Use: A Review, Anal. Chem. 88(2016)250-261.

[12] B. Gao, C. Bi, L. Fan, Designing and preparation of cytisine alkaloid surface-imprinted material and its molecular recognition characteristics, Appl. Surf. Sci. 332(2015)430-439.

[13] Y. Liu, S. Fang, J. Zhai, M. Zhao, Construction of antibody-like nanoparticles for selective protein sequestration in living cells, Nanoscale. 7(2015)7162-7167.

[14] X. Guan, X. Zhu, B. Yu, T. Zhao, H. Zhang, Preparation of temperature sensitive molecularly imprinted polymer coatings on nickel foam for determination of ofloxacin in Yellow River water by solid-phase microextraction, RSC Adv. 5(2015)91716-91722.

[15] L. Tang, C. Zhao, X Wang, R. Li, J. Yang, Y. Huang, Z. Liu, Macromolecular crowding of molecular imprinting: A facile pathway to produce drug delivery devices for zero-order sustained release, Int. J. Pharm. 496(2015)822-833.

[16] H. Hashemi-Moghaddam, S. Kazemi-Bagsangani, M. Jamili, S. Zavareh, Evaluation of magnetic nanoparticles coated by 5-fluorouracil imprinted polymer for controlled drug delivery in mouse breast cancer model, Int. J. Pharm. 497(2016)228-238.

[17] L. Zhang, Z. Qi, Q. Huang, K. Zeng, X. Sun, J. Li, Y. Liu, Imprinted-like biopolymeric micelles as efficient nanovehicles for curcumin delivery, Colloids Surf., B. 123(2014)15-22.

[18] K. Cetin, A. Denizli, 5-Fluorouracil delivery from metal-ion mediated molecularly imprinted cryogel discs, Colloids Surf., B. 126(2015) 401-406.

[19] R. Agarwal, V. Singh, P. Jurney, L. Shi, S. Sreenivasan, K. Roy, Scalable Imprinting of Shape-Specific Polymeric Nanocarriers Using a Release Layer of 
Switchable Water Solubility, ACS Nano. 6(2012) 2524-2531.

[20] A. Ruela, E. Figueiredo, G. Pereira, Molecularly imprinted polymers as nicotine transdermal delivery systems, Chem. Eng. J. 248 (2014) 1-8.

[21] D. He, S. Wang, L. Lei, Z. Hou, P. Shang, X. He, H. Nie, Core-shell particles for controllable release of drug, Chem. Eng. Sci. 125(2015)108-120.

[22] K. Deshmukh, Y. Tanwar, P. Shende, R. Cavalli, Biomimetic estimation of glucose using non-molecular and molecular imprinted polymer nanosponges, Int. J. Pharm. 494(2015)244-248.

[23] Z. Xu, D. Wang, S. Xu, X. Liu, X. Zhang, H. Zhang, Preparation of a Camptothecin Prodrug with Glutathione-Responsive Disulfide Linker for Anticancer Drug Delivery, Chem.-Asian J. 9(2014)199-205.

[24] L. Pan, Q. He, J. Liu, Y. Chen, M. Ma, L. Zhang, J. Shi, Nuclear-targeted drug delivery of TAT peptide-conjugated monodisperse mesoporous silica nanoparticles, J. Am. Chem. Soc. 134(2012)5722-5725.

[25] D. Fan, N. Li, H. Ma, Y. Li, L. Hu, B. Du, Q. Wei, Electrochemical immunosensor for detection of prostate specific antigen based on an acid cleavable linker into MSN-based controlled release system, Biosens. Bioelectron., 85(2016) 580-586.

[26] S. Liao, W. Zhang, W. Long, D. Hou, X.Yang, N.Tan, Adsorption characteristics, recognition properties, and preliminary application of nordihydroguaiaretic acid molecularly imprinted polymers prepared by sol-gel surface imprinting technology, Appl. Surf. Sci., 364(2016)579-588.

[27] D. Wang, Z. Xu, Z. Chen, X. Liu, C. Hou, X. Zhang, H. Zhang, Fabrication of Single-Hole Glutathione-Responsive Degradable Hollow Silica Nanoparticles for Drug Delivery, ACS Appl. Mater. Interfaces. 6(2014)12600-12608. 


\section{Captions}

Fig. 1 Synthesis routes of the imprint polymer nanocarrier

Fig. 2 Fluorescence spectra of the materials and DOX

Fig. 3 TEM (A) and DLS (insert in A) of MSNs and HETEM of MSNs (B) and MSN@NIP(C)

Fig. 4 Release curves of DOX from the nanocarrier under different conditions

Fig.5 Effect of MSNs@NIP (A), DOX@MSNs@MIP and DOX (B) on viability of Tca8113 cells after $24 \mathrm{~h}$ culture.

Fig. 6 CLSM pictures from Tca8113 cells incubated with DOX (a,b) and DOX@MSNs@MIP (c,d) for 3 h (a,c) and 24 h (b,d). From left to right: DAPI image, DOX image, Merge. 
1. $\mathrm{MSNs} \stackrel{\text { APTMS }}{\longrightarrow}$ MSNs-NH

2. $\mathrm{MSNs}_{-} \mathrm{NH}_{2} \underset{\mathrm{BTC}}{\longrightarrow} \mathrm{MABHD}$ MSABHD

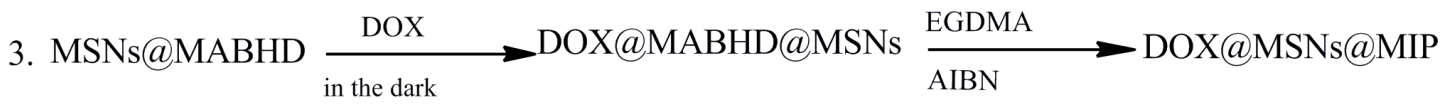

Fig 1

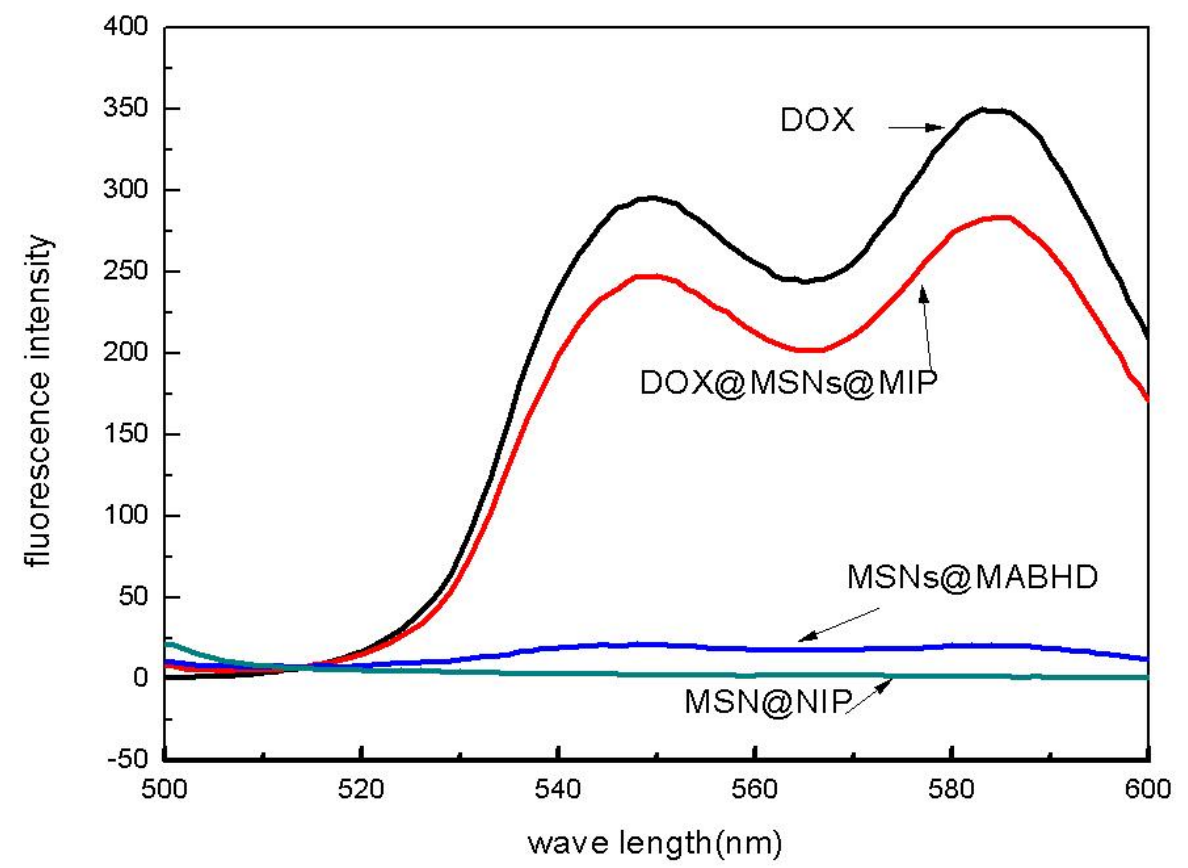

Fig2 

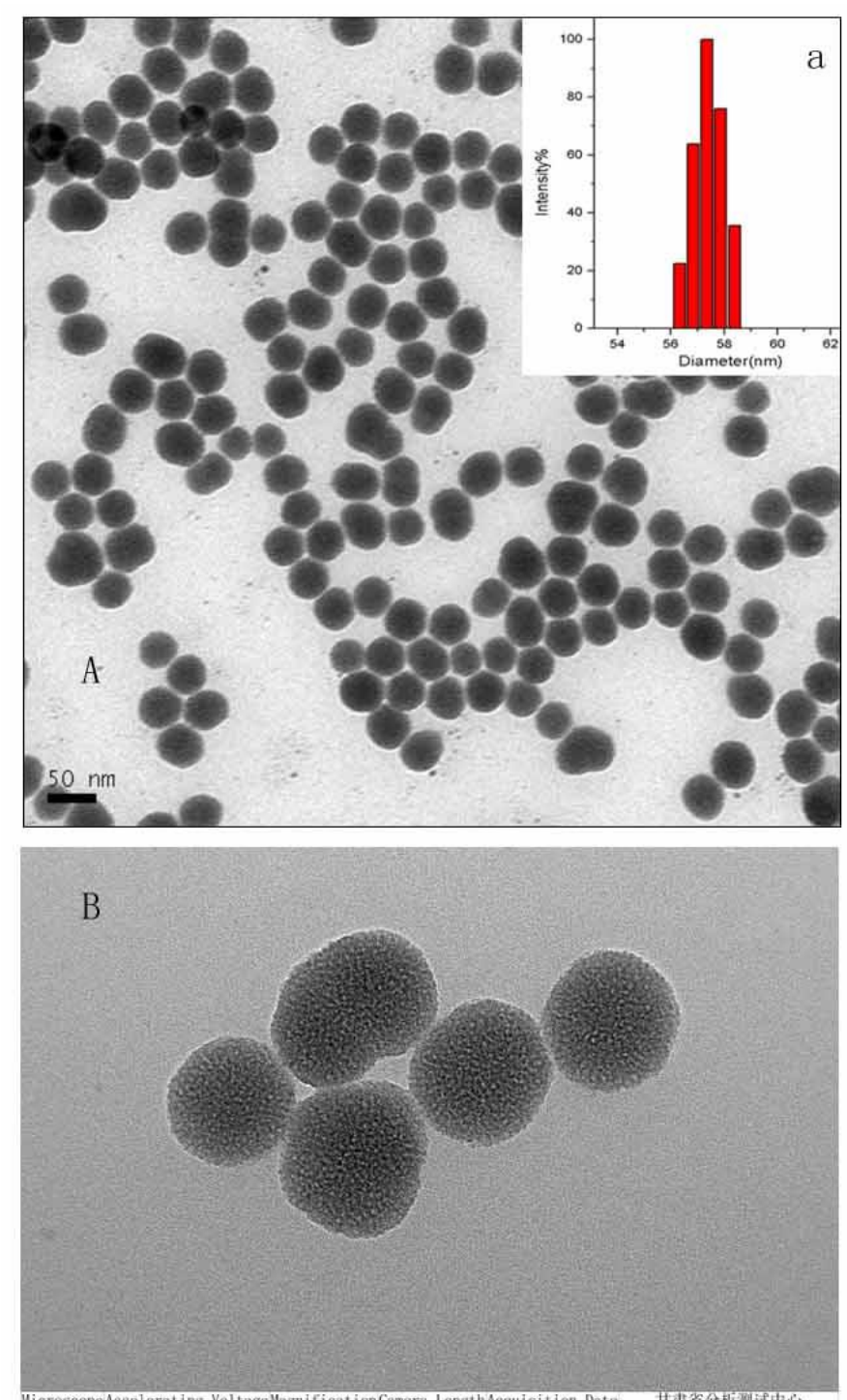

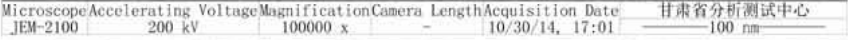

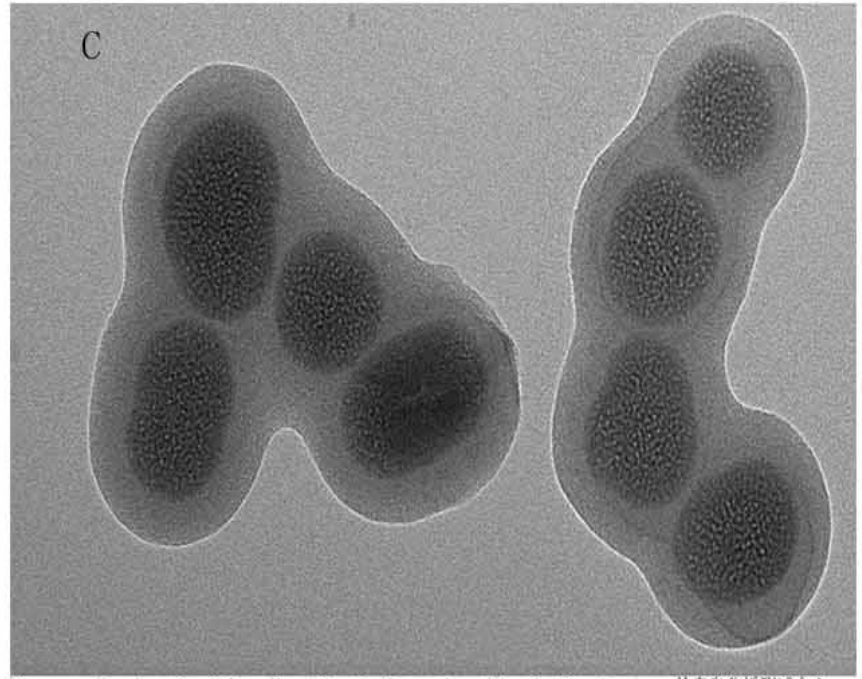

Wicroscope Accelerating Voltage VagnificicationCanera Length hcquisition Date

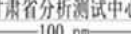

Fig 3 


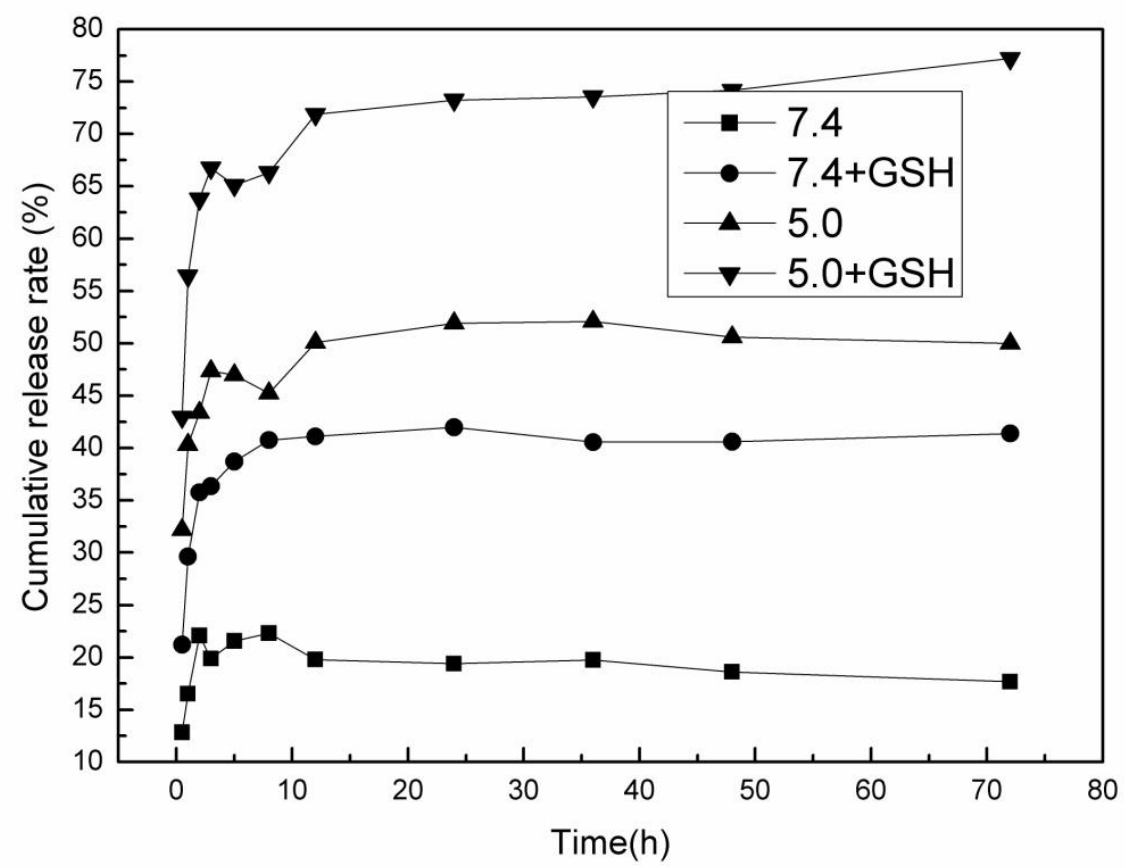

Fig4
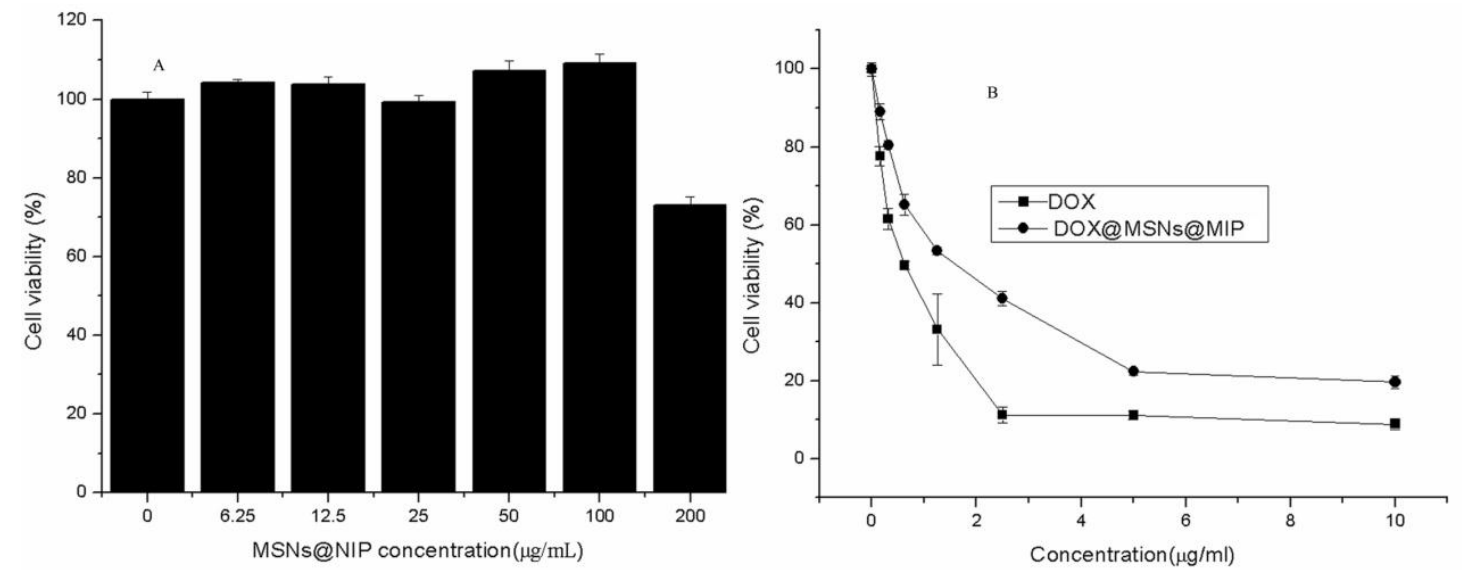

Fig5 


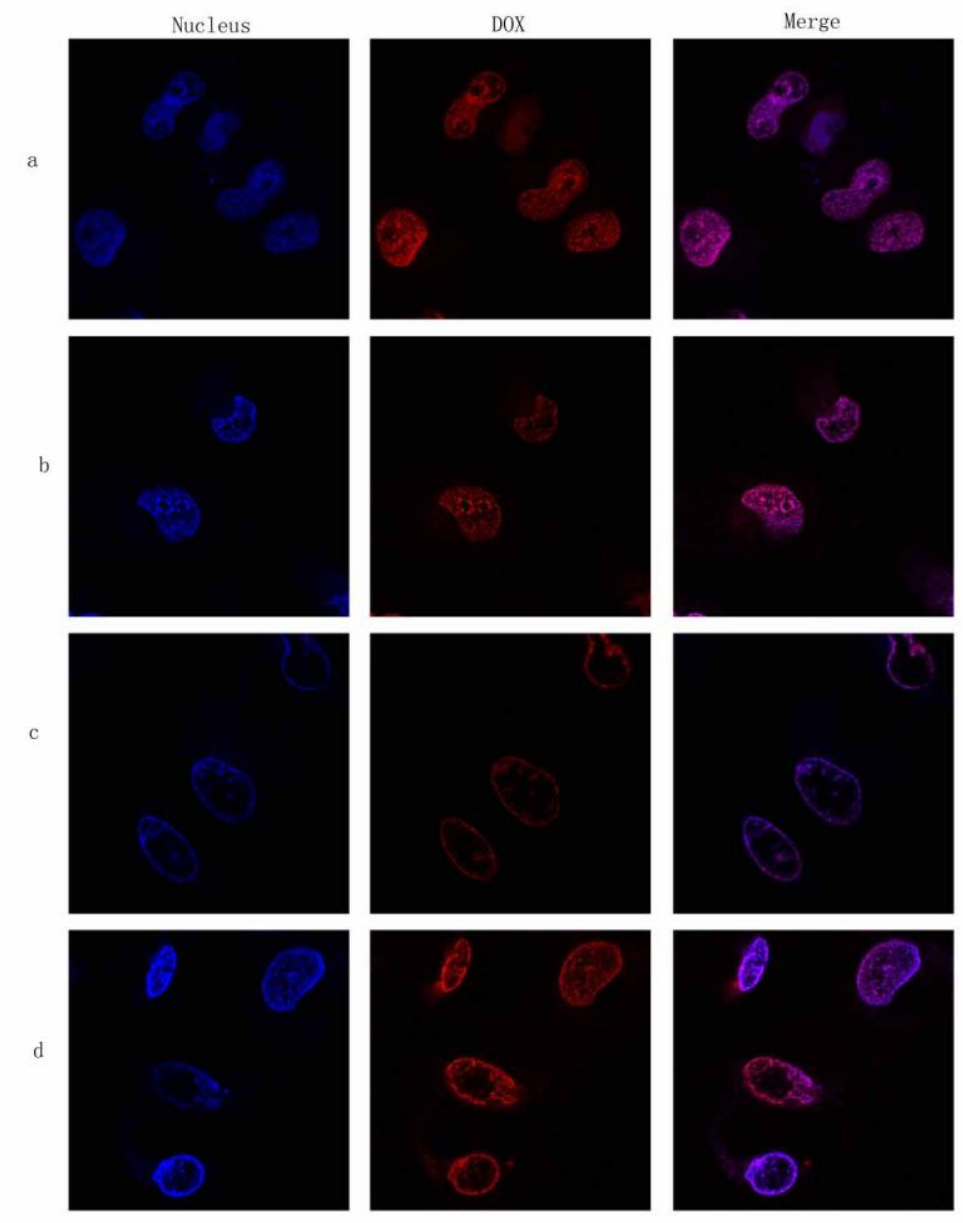

Fig6 
Table 1 Elemental analysis of MSNs, MSNs@MABHD and MSN@NIP

\begin{tabular}{lllll}
\hline materials & $\mathrm{N}(\%)$ & $\mathrm{C}(\%)$ & $\mathrm{H}(\%)$ & $\mathrm{S}(\%)$ \\
\hline MSNs & 0 & 1.025 & 0.602 & - \\
MSNs@MABHD & 0.805 & 5.545 & 1.388 & 0.694 \\
MSNs@NIP & 0.825 & 42.60 & 4.984 & 0.636 \\
\hline
\end{tabular}




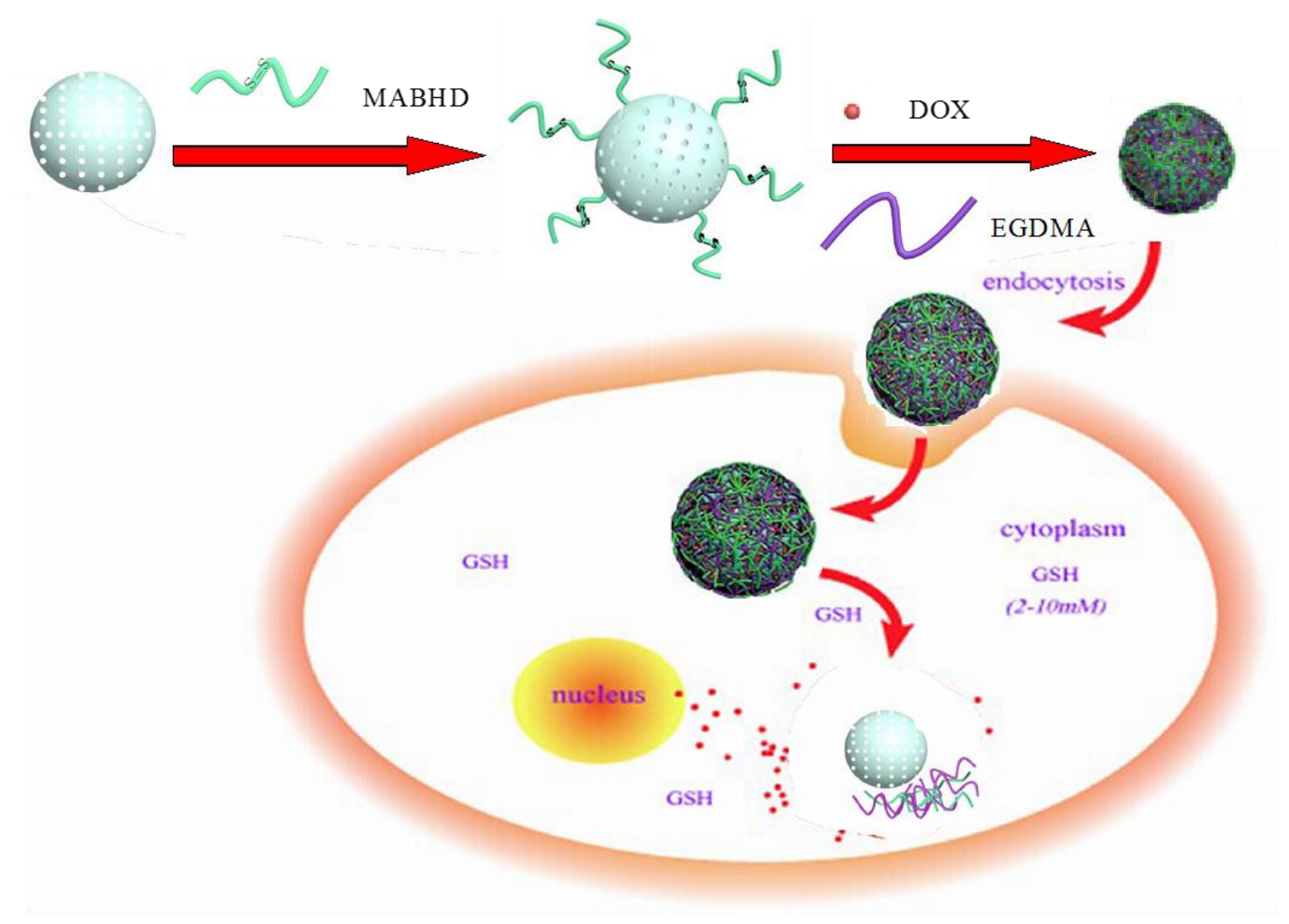

